\title{
Factors associated with statin-related adverse muscular events in adult dyslipidemic outpatients
}

\author{
Paula Ferreira Castro ${ }^{1 *}$, Eliane Ribeiro ${ }^{2,3}$, Egidio Lima Dorea ${ }^{3}$, Gelba Almeida Pinto ${ }^{3}$, \\ Rosario Dominguez Crespo Hirata ${ }^{1}$
}

\begin{abstract}
${ }^{1}$ Department of Clinical and Toxicological Analyses, School of Pharmaceutical Sciences, University of São Paulo, Sao Paulo, SP, Brazil, '2 Department of Pharmacy, School of Pharmaceutical Sciences, University of Sao Paulo, São Paulo, SP, Brazil,

${ }^{3}$ University Hospital, University of Sao Paulo, São Paulo, SP, Brazil
\end{abstract}

\begin{abstract}
Statins are the most prescribed lowering-cholesterol drugs. They are well tolerated, however, some patients present muscular adverse symptoms. Clinical and laboratory data from 120 dyslipidemic patients prescribed with statins were obtained from January to December/2013 at a University Hospital in Sao Paulo city, Brazil, to study factors associated with statin-related adverse muscular events (AME). Pharmacotherapy and statin-related AME data (serum CK elevation and any degree of myopathy, myalgia, myositis or rhabdomyolysis) of the dyslipidemic patients were recorded. The study was approved by local Ethics Committees. Simvastatin $(70 \%)$ and atorvastatin $(25 \%)$ were the most prescribed statins. AME related to statin treatment were found in $17 \%$ of the patients. Mean age and use of simvastatin were lower in AME group than non-AME group $(\mathrm{p}<0.05)$. Simvastatin users were less likely to develop AME than atorvastatin users $(\mathrm{OR}=0.21 ; 95 \% \mathrm{CI}=0.07-0.57 ; \mathrm{p}<0.01)$. The use of $\mathrm{P}$-glycoprotein $(\mathrm{ABCB} 1)$ efflux pump inhibitors was associated with high risk for $\mathrm{AME}(\mathrm{OR}=5.26 ; 95 \% \mathrm{CI}=1.55-17.79 ; \mathrm{p}<0.01)$. Serum liver enzymes were increased up to three-fold in $2.5 \%$ of the statin-treated patients. The results are suggestive that the type of statin prescribed and the concomitant use of ABCB1 inhibitors increase the susceptibility to adverse muscular events during statin therapy in dyslipidemic outpatients.
\end{abstract}

Keywords: Statins/study/effects. Statins/muscular adverse event. Dyslipidemia. Drug intolerance. Drug interaction/pharmacotherapy.

\section{INTRODUCTION}

Dyslipidemia is a disorder in lipid metabolism that results in changes in blood concentrations of lipids, mainly cholesterol and triglycerides. The presence of dyslipidemia is closely linked to increased cardiovascular risk. Mortality from coronary artery disease is the leading cause of death in the country, and there is evidence pointing high cholesterol as its main risk factor (Institute of Medicine, 2010; Xavier et al., 2013).

Statins are a validated therapy for dyslipidemia control and for reducing the incidence of cardiovascular events (Xavier et al., 2013). They are reversible inhibitors of the enzyme hydroxymethylglutaryl coenzyme A (HMG$\mathrm{CoA}$ ) reductase, a key enzyme of the cholesterol synthesis

\footnotetext{
*Correspondence: P. F. Castro. Faculdade de Ciências Farmacêuticas, Universidade de São Paulo. Av. Prof. Lineu Prestes, 580. B-17, 05508-000 - São Paulo, SP, Brasil. Tel: +55 11 99656-9599. E-mail: paula.castro@usp.br
}

pathway, decreasing intracellular production of cholesterol and, consequently, cholesterolemia. Statins are considered very effective in reducing morbidity and mortality of the cardiovascular disease in high risk patients (Minder et al., 2013).

The V Brazilian Guidelines for Dyslipidemia Treatment, currently followed by clinicians in Brazil, elects statins as the first line of treatment for primary and secondary cardiovascular prevention therapies, starting with low doses and increasing progressively as needed (Xavier et al., 2013).

Statins differ in molecular and pharmacokinetic characteristics such as lipophilicity, half-life and rate of protein binding, which influence their dosage, efficacy and safety. Statins are metabolized by the cytochrome P450 (CYP450) isoenzyme system, excepting pravastatin, which is transformed enzymatically by sulfotransferases in the liver cytosol (Jacobsen et al., 1999). The CYP3A4 isoenzyme is particularly important in the metabolism 
of atorvastatin and simvastatin and may lead to drug interactions with other drugs. Since fluvastatin and rosuvastatin are mainly metabolized by CYP2C9, and pravastatin is metabolized by CYP3A4 to a lesser extent, they have smaller potential for drug interaction (Armitage, 2007).

Other important factors for statin bioavailability are the membrane transporters P-glycoprotein (ABCB1) and organic anion-transporting polypeptide OATP1B (Holtzman et al., 2006; Kalliokoski, Niemi, 2009). OATPB1 promotes uptake and ABCB1 promotes the efflux of statins in the intestine and liver. Therefore, drug interactions involve differences in expression not only of CYP450 isoenzymes, but also in ABCB1 and OATPB1 (Holtzman et al., 2006; Kalliokoski, Niemi, 2009; Link et al., 2008).

Studies have shown that 5 to $10 \%$ of patients are intolerant to statins (Eckel, 2010; Mammen, Amato, 2010; Oh et al., 2007). Intolerance to statins is often related to muscle symptoms such as muscle pain, elevated serum levels of creatine kinase (CK) and rhabdomyolysis (Reiner, 2014)

The American College of Cardiologists (ACC) and the American Heart Association (AHA) have defined four terms for adverse muscular events (AME): (i) myopathy: any muscle complaint; (ii) myalgia: muscle complaints without elevation in CK levels; (iii) myositis: muscle complaints along with serum CK elevation; (iv) rhabdomyolysis: extreme elevation in CK levels (10-fold or more higher than the upper limit of normality) (Ahmad, 2014).

Since hypercholesterolemia is often asymptomatic, any unpleasant effect can lead to treatment abandonment. Monitoring and preventing AME, therefore, is essential to ensure proper adherence to the pharmacotherapy, to reduce cardiovascular risk and to improve the quality of life of the patients.

We have investigated the safety profile of statins in adult dyslipidemic outpatients attending the Metabolic Diseases Service at the University Hospital of the University of São Paulo (HU/USP), in order to study factors associated with statin-related AME.

\section{SUBJECTS AND METHODS}

\section{Subjects and study design}

This is a non-interventionist cross-sectional study that took place at the Metabolic Diseases Service of the HU/USP, a service formed by a multidisciplinary team that provides health care for adult outpatients with diabetes, hypertension and dyslipidemia.
The study investigated the safety profile of statin treatment in adult outpatients attended at this service from January to December/2013. The study was approved by local Ethics Committees from the School of Pharmaceutical Sciences of the University of São Paulo (protocol \#697.094) and HU/USP (protocol \#728.231).

The study included patients with dyslipidemia who had statins prescribed and had lipid profile results from 2013 available at the hospital electronic system. Patients without complete recorded data, who had been hospitalized or immobilized in the month before the study, who had suffered any fracture or prolonged surgery (hip fracture), convulsive patients, patients with muscle diseases and patients who had suffered acute myocardial infarction the month before the analysis were excluded.

The sample size was calculated considering $95 \%$ of confidence level, $5 \%$ of confidence interval (alpha value) and the minimum of $10 \%$ of AME related to statin therapy (statin intolerance) according to previous published data (Bitzur et al., 2013; Eckel, 2010; Mammen, Amato, 2010; Oh et al., 2007). One hundred and twenty out of 692 dyslipidemic patients attended in 2013 were randomly selected.

Patient information that could contribute to statin intolerance, such as gender, age, weight, height, presence of comorbidities, use of concomitant medications, type of statin used, physical activity practice, alcohol intake and tobacco smoking were obtained from medical records. Laboratory data to assess liver function, kidney function, diabetes mellitus, thyroid dysfunction and muscle injury were obtained from the electronic system available at the service.

Laboratory data included triglycerides, alanine aminotransferase (ALT), aspartate aminotransferase (AST), gamma-glutamyl transferase, urea, creatinine, glucose, glycated hemoglobin, thyroid stimulating hormone, and CK. The reference values of CK were 32 to $294 \mathrm{U} / \mathrm{L}$ for men and 33 to $211 \mathrm{U} / \mathrm{L}$ for women. Reference values for AST were 10 to $35 \mathrm{U} / \mathrm{L}$ for men and 10 to 31 U/L for women. Reference values for ALT were 9 to 43 $\mathrm{U} / \mathrm{L}$ for men and 9 to $36 \mathrm{U} / \mathrm{L}$ for women.

The cut-off for age was set at 60 years old, which is the age used to classify elderly subjects in Brazil, according to the Brazilian Institute of Geography and Statistics (http://www.ibge.gov.br). As physical activity we considered the practice of regular exercise for at least 150 minutes per week.

Pharmacological therapies were recorded. The medicines used by the patients were listed and classified according to their ability to induce, to inhibit or to be 
substrate of CYP450 isoenzymes (Lexicomp, 2014) and ABCB1 (Holtzman et al., 2006). The prescription of 5 or more drugs was considered polypharmacy (Carvalho et al., 2012).

The level of interactions was evaluated using the Micromedex ${ }^{\circledR}$ Drug Interaction tool (Truven Health Analytic, 2015). According to Micromedex ${ }^{\circledR}$ database, drug interactions are classified as contraindicated associations, major, moderate and minor interactions. Major interactions may be life-threatening and/or require medical intervention to minimize or prevent serious adverse effects. Changes in therapy should be considered. Moderate interactions may result in exacerbation of the patient's condition and may require a change in treatment. Minor interactions result in limited clinical effects, usually requiring no change in therapy (Truven Health Analytic, 2015).

AME in statin-treated patients were identified based on medical records of physical examinations or patient's complaints and clinical laboratory tests. Serum CK elevation and any degree of myopathy, myalgia, myositis or rhabdomyolysis were considered AME.

\section{Statistical analysis}

The results were analyzed using Open Epi software version 3.03a (Dean, Sullivan, Soe, 2015). Missing data were excluded from the analysis. Continuous variables are expressed as mean $\pm \mathrm{SD}$ and were compared by $t$-test. Categorical variables are expressed as percentage and were compared by Chi-square test or Fisher's exact test. The risk of AME to statin treatment was assessed by Odds ratio (OR) and 95\% confidence interval (CI95\%) estimates. Accepted significance level was $p<0.05$ for two-sided tests. Figures were constructed using Graph Pad Prism 5.0 software (GraphPad Software Inc., La Jolla, CA, USA).

\section{RESULTS}

\section{Clinical and laboratory data}

Demographic and baseline characteristics for patients assessed in this study are presented in Table I. The mean age of the total sample group was $60.9 \pm 11.2$ years old. Out of the 120 dyslipidemic patients, 21 (17\%) had AME. Mean age was lower in the AME group than in the non-AME group $(\mathrm{p}<0.01)$.

The prevalence of known risk factors for adverse events such as gender, BMI, physical activity, smoking, alcohol consumption, associated diseases and the use of concomitant medications (polypharmacy) was similar among patients with and without adverse events related to statins (Table I, $\mathrm{p}>0.05$ ).

A three-fold increase in serum liver enzymes during statin therapy was found in $2.5 \%$ of the patients. This adverse event did not differ in AME and non-AME patients (data not shown).

\section{Statin therapy and adverse events}

The relation between the manifestation of AME and the type of statin used was also investigated. The most frequently used statin was simvastatin (70\%), followed by atorvastatin (25\%) (Table I). Rosuvastatin and pravastatin were less used (4\% and $1 \%$, respectively) probably due to economic difficulties in acquiring them. Therefore, statistical analyzes were performed comparing only simvastatin with atorvastatin. It was observed that simvastatin users were less likely to develop AME than users of atorvastatin $(\mathrm{OR}=0.21 ; \mathrm{CI} 95 \%=0.07-0.57$; $\mathrm{p}<0.01$ ) (Table I, Figure 1). The prevalence of myalgia was higher in patients taking atorvastatin $(23 \%)$ than in those using simvastatin ( $2 \%)$, but the difference was not statistically significant ( $\mathrm{p}=0.28$; data not shown).

In 17 cases, statin therapy was suspended (11 therapies with simvastatin suspended, 4 with atorvastatin and 2 with rosuvastatin), and in 18 cases the statin used was replaced by another statin. There was no explanation in the medical records for the suspension or the switch in 20 of the 35 cases (57\%). It is possible that in two of the cases simvastatin was replaced by atorvastatin aiming better control of dyslipidemia, since LDL cholesterol values were elevated $(>160 \mathrm{mg} / \mathrm{dL})$. Considering the remaining 15 cases, the therapy was altered in $12(34 \%)$ because of AME, in two (6\%) because of elevated liver enzymes and in one $(3 \%)$ as an attempt to increase adherence to treatment (Figure 2).

Therapy usually starts with simvastatin, which, in case of adverse events or therapy failure, may be replaced by a more powerful statin or one less likely to cause adverse events (Bitzur et al., 2013; Bruckert et al., 2005). Only once no report of previous use of simvastatin was found. In this case, atorvastatin was replaced by rosuvastatin without explanation. Simvastatin was replaced by atorvastatin in 13 cases and by rosuvastatin in two cases. One patient originally used simvastatin, which was changed to pravastatin because of leg pain, then replaced by ezetimibe due to headache and dizziness; since there was no adherence to treatment, it was finally changed to atorvastatin. In one other case, simvastatin was also replaced by pravastatin due to AME, and later changed to rosuvastatin without further explanation (Figure 3). 
TABLE I - Demographic and clinical characteristics of dyslipidemic outpatients

\begin{tabular}{|c|c|c|c|c|}
\hline Variables & Total (120) & Non-AME (99) & AME (21) & p-value \\
\hline Age, years & $60.9 \pm 11.2$ & $62.1 \pm 10.8$ & $55.5 \pm 12.2$ & 0.01 \\
\hline \multicolumn{5}{|l|}{ Gender } \\
\hline Female & $56 \%(67)$ & $79 \%(53)$ & $21 \%(14)$ & 0.27 \\
\hline Male & $44 \%(53)$ & $87 \%(46)$ & $13 \%(7)$ & \\
\hline \multicolumn{5}{|l|}{ Age groups } \\
\hline$\leq 59$ years & $48 \%(58)$ & $76 \%(44)$ & $24 \%(14)$ & 0.06 \\
\hline$\geq 60$ years & $52 \%(62)$ & $89 \%(55)$ & $11 \%(7)$ & \\
\hline Type 2 Diabetes & $63 \%(76)$ & $84 \%(64)$ & $16 \%(12)$ & 0.52 \\
\hline Hypertension & $65 \%(78)$ & $81 \%(63)$ & $19 \%(15)$ & 0.49 \\
\hline Obesity & $32 \%(39)$ & $87 \%(34)$ & $13 \%(5)$ & 0.34 \\
\hline BMI, kg/m² & $29.2 \pm 5.4$ & $29.3 \pm 5.7$ & $28.6 \pm 3.9$ & 0.54 \\
\hline Hypothyroidism & $13 \%(16)$ & $94 \%(15)$ & $6 \%(1)$ & $0.36^{a}$ \\
\hline Chronic kidney disease & $5 \%(6)$ & $50 \%(3)$ & $50 \%(3)$ & $0.13^{a}$ \\
\hline Liver Failure & $2 \%(3)$ & $67 \%(2)$ & $33 \%(1)$ & 0.88 \\
\hline Tobacco smoking & $27 \%(32)$ & $78 \%(25)$ & $22 \%(7)$ & 0.29 \\
\hline Alcohol intake & $4 \%(5)$ & $100 \%(5)$ & $0 \%(0)$ & $0.71^{a}$ \\
\hline Physical Activity & $47 \%(57)$ & $77 \%(44)$ & $23 \%(13)$ & 0.18 \\
\hline Total cholesterol, mg/dL & $185 \pm 43$ & $182 \pm 40$ & $202 \pm 57$ & 0.16 \\
\hline LDL cholesterol, mg/dL & $103 \pm 36$ & $101 \pm 34$ & $112 \pm 44$ & 0.30 \\
\hline HDL cholesterol, mg/dL & $52 \pm 18$ & $51 \pm 15$ & $55 \pm 31$ & 0.56 \\
\hline Polypharmacy & $50 \%(60)$ & $85 \%(51)$ & $15 \%(9)$ & 0.47 \\
\hline \multicolumn{5}{|l|}{ Statin therapy } \\
\hline Simvastatin & $70 \%(84)$ & $89 \%(75)$ & $11 \%(9)$ & 0.001 \\
\hline Atorvastatin & $25 \%(30)$ & $63 \%(19)$ & $37 \%(11)$ & \\
\hline Others (rosuvastatin, pravastatin) & $5 \%(6)$ & $83 \%(5)$ & $16 \%(1)$ & \\
\hline
\end{tabular}

Number of individuals are shown in parentheses. AME: adverse muscular event; BMI: body mass index; LDL: low density lipoprotein; HDL: high density lipoprotein. Continuous variables are shown as mean \pm standard deviation and compared by $t$-test. Categorical variables were compared by chi-square test or Fisher test $\left.{ }^{(a}\right)$.

\section{Drug interactions with statins}

Patients used an average of 3.8 (range 1 to 11 ) therapy drugs. Only 8 patients $(7 \%)$ used statin as monotherapy, 52 patients (43\%) were prescribed 2 to 4 drugs and $60(50 \%)$ patients had prescription of 5 or more drugs. The use of concomitant medications, including polypharmacy (Table I) did not differ in AME e non-AME groups $(\mathrm{p}<0.05)$.

The most commonly used drugs were metformin (44\%), acetylsalicylic acid (38\%), hydrochlorothiazide (33\%), enalapril (22\%), losartan (22\%), amlodipine (22\%) and omeprazole (19\%).

We also studied the interaction between AME and concomitant medications, which interact via CYP2C9,
CYP3A4 and ABCB1. Sixty-five percent $(n=78)$ of the patients used at least one drug with potential to interact with the statin, and 15 of these (19\%) had an AME. The concomitant use of ABCB1 inhibitors was associated to the onset of $\mathrm{AME}(\mathrm{OR}=5.26, \mathrm{CI} 95 \%=1.55-17.79 ; \mathrm{p}<0.01)$, however no interactions were found with CYPs (Figure 1).

Major interactions with statins found in the therapy of the patients are shown in Table II. Even though it is not possible to associate the use of drugs that interact with statin and the presence of adverse events, the patient who used itraconazole with atorvastatin had increased CK levels and the patient who used diltiazem had simvastatin switched to pravastatin because of ALT elevation, with improvement of the clinical condition after simvastatin suspension. Unfortunately, the reason for switching 
Odds Ratio $(95 \% \mathrm{CI}) \quad$ p-value

\begin{tabular}{ll}
\hline $1.42(0.55-3.66)$ & 0.47 \\
$0.35(0.07-1.60)$ & 0.26 \\
$\mathbf{5 . 2 6}(\mathbf{1 . 5 5}-\mathbf{1 7 . 7 9})$ & $<\mathbf{0 . 0 1}$ \\
$1.01(0.36-2.88)$ & 0.98 \\
$2.07(0.69-6.16)$ & 0.18 \\
$1.08(0.41-2.84)$ & 0.88 \\
$\mathbf{0 . 2 1}(\mathbf{0 . 0 7}-\mathbf{0 . 5 7})$ & $<\mathbf{0 . 0 1}$
\end{tabular}

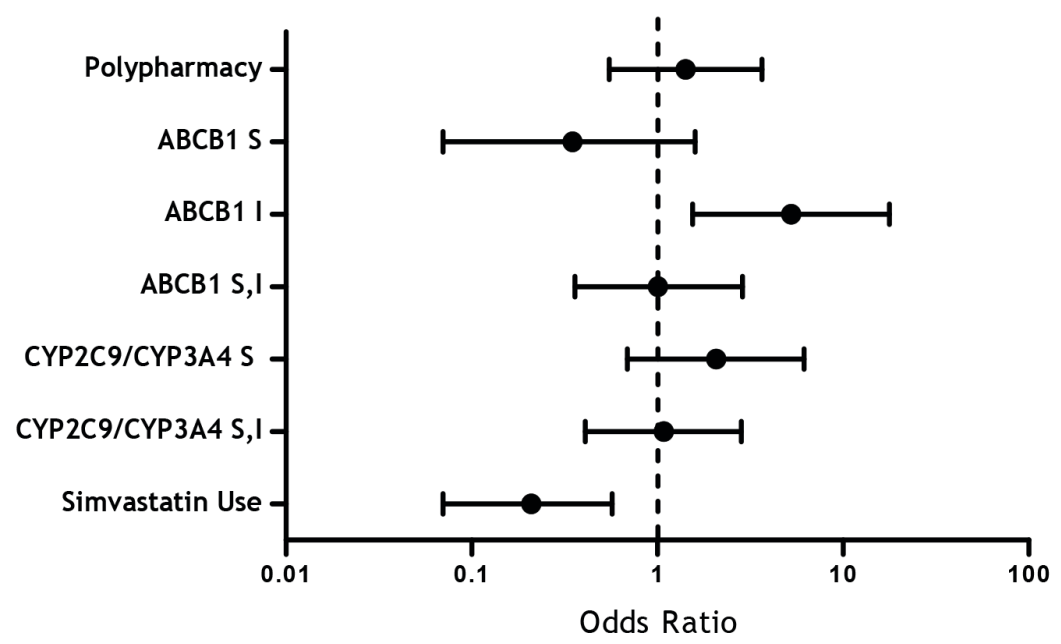

FIGURE 1 - Pharmacotherapy factors associated with statin-related adverse muscular events in dyslipidemic patients. ABCB1: P-glycoprotein; S: Substrate; I: Inhibitor; CI: confidence interval.

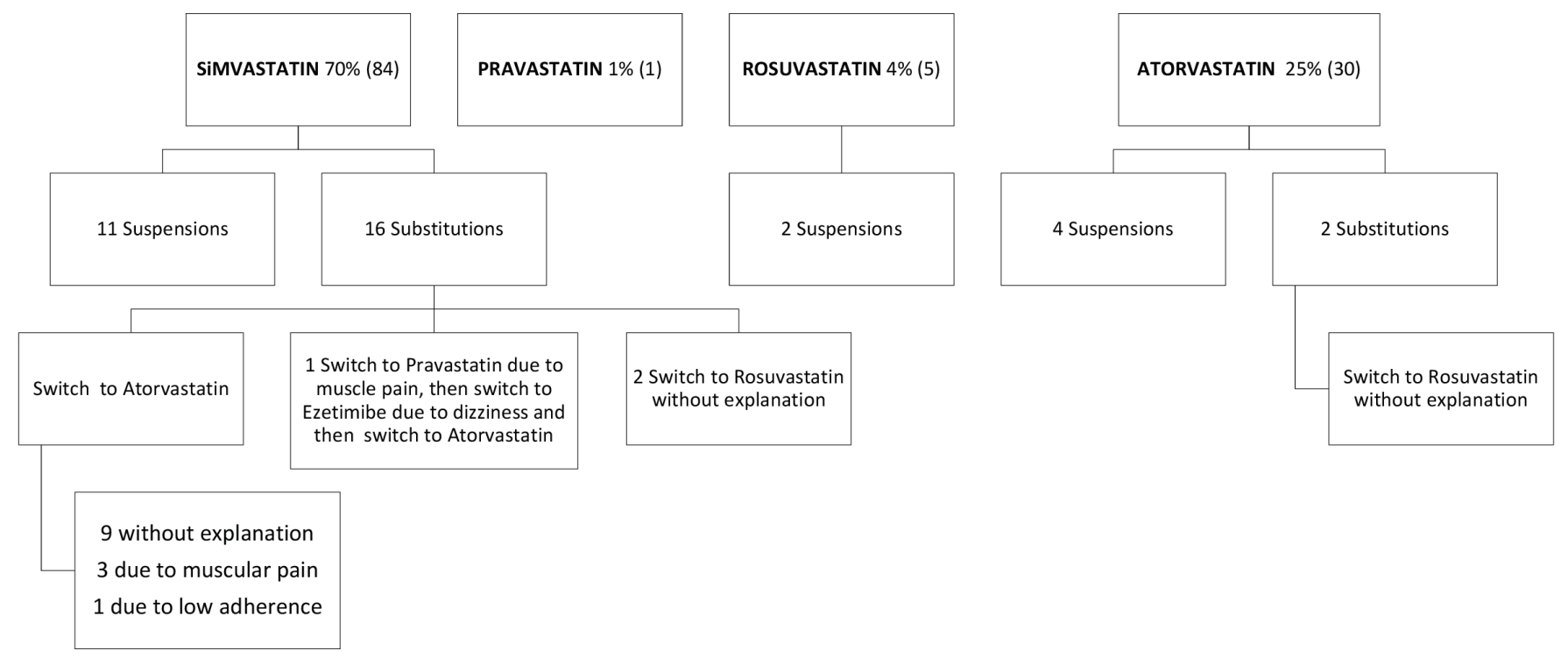

FIGURE 2 - Schematic diagram illustrating statin therapy suspensions and substitutions in dyslipidemic outpatients.

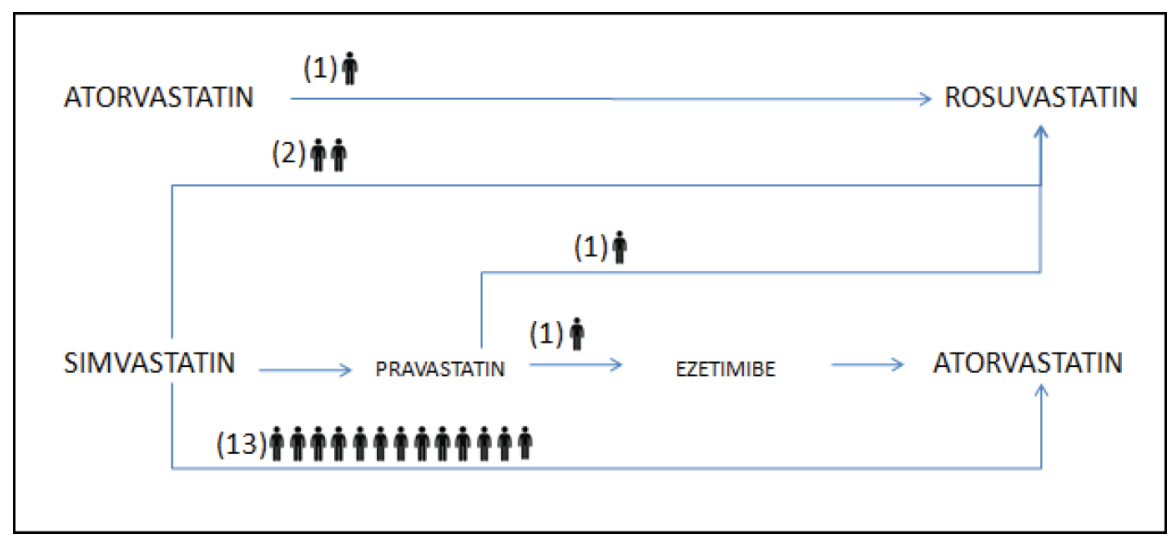

FIGURE 3 - Schematic diagram illustrating statin therapy substitutions in dyslipidemic patients. 
TABLE II - Major interactions between statins and therapy drugs used by dyslipidemic patients

\begin{tabular}{llll}
\hline Number of Patients & Statin Used & Drug Associated & Clinical Result \\
\hline 1 & Atorvastatin & Itraconazole & CK elevation, with no muscular complaints \\
Simvastatin & Simvastatin switched to atorvastatin, with no \\
1 & Simvastatin & Diltiazem & $\begin{array}{l}\text { Simvastatin switched to pravastatin due to ALT } \\
\text { elevation, with improvement of the clinical } \\
\text { condition after simvastatin suspension }\end{array}$ \\
1 & Simvastatin & Amiodarone & $\begin{array}{l}\text { No adverse events } \\
\text { No adverse events }\end{array}$ \\
3 & Simvastatin & Fenofibrate/ Amlodipine & Liver enzyme elevation without change in therapy \\
\hline
\end{tabular}

Level of interactions evaluated using the Micromedex ${ }^{\circledR}$ Drug Interaction tool. Major interactions: may be life-threatening and/or require medical intervention to minimize or prevent serious adverse effects (Truven Health Analytic, 2015).

statins in the patient who used warfarin, diltiazem and simvastatin concomitantly is not known, but the presence of several risk factors is suggestive that adverse events have occurred.

\section{DISCUSSION}

The study group was homogeneous in relation to gender and had a mean age of 60.9 years, which is expected, since the prevalence of metabolic diseases increases with age (IBGE, 2013). The prevalence of diabetes, obesity and hypertension was high. These characteristics are associated with increased risk of cardiovascular disease, even though nearly half of the patients have declared to practice some physical activity, which is a protective factor for that.

In this study, $17 \%$ of dyslipidemic patients had AME associated to the use of statins. Other studies with dyslipidemic patients treated with statins reported muscle events incidences of 5 to 10\% (Ahmad, 2014; Bruckert et al., 2005; Eckel, 2010; Oh et al., 2007) that may be even higher when considering only myalgia (9 to 20\%) (Mammen, Amato, 2010). The differences in AME prevalence among studies may be due to the specific criteria, such as muscle pain or slight elevation of CK (Mammen, Amato, 2010).

The mechanisms by which statins cause muscle damage are not well understood. Studies have shown that statins, by blocking the mevalonate synthesis pathway, decrease ubiquinone (CoQ10) production, an end product of mevalonate pathway, which is part of the mitochondrial electron transport system (oxidative phosphorylation), responsible for producing energy to muscle cells. A reduction in CoQ10 could lead to mitochondrial dysfunction and myopathy (Bitzur et al., 2013). It is also likely that the reduction of other end products of the mevalonate pathway, such as farnesyl pyrophosphate and geranylgeranyl pyrophosphate, may play a role in statin-induced myopathy, since they normally activate regulatory proteins that promote cell growth and maintenance and reduce apoptosis (Bitzur et al., 2013). Another hypothesis is that the reduction of the cholesterol content in skeletal muscle cells membranes makes them unstable and alters ionic permeability, affecting membrane excitability (Bitzur et al., 2013).

In this study, physical activity practice has not increased the predisposition to AME. The practice of heavy or excessive exercise is well described as a risk factor for the onset of muscular events (Ahmad, 2014; Bruckert et al., 2005), since it predisposes to muscle injury, probably by inflammatory mechanism (Mancini et al., 2013).

AME patients had lower mean age in comparison with non-AME group, in this work. It is expected that elderly people are more likely to have drug-related adverse events, since they usually have several comorbidities, use polypharmacy and have physiological changes that affect the metabolism of drugs (Shi, Klotz, 2011). In the PRIMO study, Bruckert et al. (2005) found that symptomatic patients were more active, probably because they were able to keep the type of muscle damage that is exacerbated by statins (Bruckert et al., 2005). A decrease in physical activity in older patients may be the reason for the lower incidence of adverse events.

Elevation of liver enzymes is observed in a small proportion of patients taking statins (3\%), and an even smaller part of these patients progress to hepatitis (Jose, 2016; Russo et al., 2014). In this study, the frequency of liver enzymes elevation $(2.5 \%)$ was similar to that of these previous studies. The liver damage mechanisms are not fully understood. It is known that the lesion may have 
hepatocellular, cholestatic or autoimmune patterns (Jose, 2016; Mancini et al., 2013; Russo et al., 2014). The most common histopathological findings in statin hepatotoxicity is portal inflammation with lymphocytes, with or without cholestasis. In autoimmune hepatitis, it is proposed that the drug acts as a hapten for cellular targets in genetically predisposed hosts who are re-exposed to statin (Russo, Scobey, Bonkovsky, 2009). Hepatotoxicity of statins is dose dependent and occurs mainly in the first months of therapy (Russo et al., 2014).

Simvastatin was the most commonly prescribed statin, probably because it is more available in the primary health assistance, while atorvastatin, the second most commonly used statin, is also available, but requires a more bureaucratic process to be acquired (Brasil, 2009; 2013). A study in basic health units in a small city of the State of São Paulo showed that simvastatin was the statin of choice in $79 \%$ of cases, followed by atorvastatin $(21 \%)$ due to its availability at the Public Health System (Bonfim et al., 2013).

Type and solubility of statin may be associated with the occurrence of adverse events (Bitzur et al., 2013). Soluble statins, such as simvastatin, seem to cause more adverse events as they penetrate cell membrane more easily (Liao, 2002). According to the PRIMO study, the most hydrophilic statins were less likely to cause myalgia, while simvastatin, the most lipophilic one, was most likely to be associated with AME (Bitzur et al., 2013; Bruckert et al., 2005).

Interestingly, a greater number of AME was detected in patients using atorvastatin (37\%) than in those using simvastatin $(11 \%)$. Similar results were reported by the study PRIMO, which showed that among patients who had muscular symptoms, $22.6 \%$ were using simvastatin and $33.1 \%$ atorvastatin (Bruckert et al., 2005). However, for patients in this study, atorvastatin was typically used as a second option, as an alternative for cases in which the patient had adverse events. Thus, these individuals had a greater predisposition to have muscular events, since they had already had them while taking simvastatin.

Considering the adverse events when choosing patient's therapy seems to be quite important, since, in our study, in $40 \%$ of cases the treatment was altered or suspended due to muscular events or elevated liver enzymes. It has been reported that $28 \%$ of statin users switched statins at some point due to adverse events (Birtcher, 2015).

Polypharmacy was detected in half the patients, which may be due to the high prevalence of comorbidities. Polypharmacy has been associated with adverse health events such as side effects, drug interactions, low adherence to treatment, hospitalization and costs related to the treatment of its complications (Viktil, Blix, Reikvam, 2008). Thus, the potential for drug interactions that may increase statin bioavailability is a relevant factor for the appearance of AME (Bellosta, Paoletti, Corsini, 2004; Thai et al., 2016). The prevalence of polypharmacy $(50 \%)$ found in our study is higher than the one found in a large study among community dwelling-elderly who were taking medicines for diabetes and cardiovascular diseases in Sao Paulo city (36\%) (Carvalho et al., 2012). This difference may be due to higher number of medicines taken by the dyslipidemic patients at our hospital, which have multiple-associated comorbidities, such as type 2 diabetes, obesity and hypertension.

At pharmacodynamics level, statins are not expected to interfere with other drugs, since they are selective inhibitors of HMG-CoA reductase and usually do not show relevant affinities toward other enzymes or receptor systems. However, at pharmacokinetics level, they have potential for drug interactions (Bellosta, Paoletti, Corsini, 2004). The use of concomitant medications which are metabolized by the same CYP450 enzyme may lead to an increase of statin concentration, exposing the patients to adverse events (Bellosta, Paoletti, Corsini, 2004).

A study done by the Food and Drug Administration (FDA) of rhabdomyolysis related to statin showed that, in 3339 reports of rhabdomyolysis, approximately $58 \%$ of the cases were associated with concomitant use of drugs that affected the metabolism of statins, including fibrates, warfarin and azole antifungals (Bellosta, Paoletti, Corsini, 2004).

In this work, $65 \%$ of the prescriptions had potential statin-other drugs interactions (data not shown). A systematic review from 2016 found that the prevalence of potential statin-drug interaction in older people ranged from 0.19 to $33.0 \%$ (Thai et al., 2016).

An association between the concomitant use of $\mathrm{ABCB} 1$ inhibitors and the risk for muscular events was found. It is likely that by inhibiting the efflux pump in the enterocytes, the absorption and circulating levels of statin increase, leading to a higher risk of muscular events (Holtzman et al., 2006).

CYP2C9 and CYP3A4 substrates or inhibitors and ABCB1 substrates can increase statin bioavailability. However, they were not a relevant factor of AME risk in this work.

Concomitant use of itraconazole, an azole antifungal, with atorvastatin is not contraindicated, but it can elevate atorvastatin plasma levels (2.5-fold) after a dose of $20 \mathrm{mg}$, thus increasing the risk of myopathy or rhabdomyolysis. This is probably due to the ability of itraconazole to 
inhibit the first-pass elimination of atorvastatin mediated by CYP3A4, which does not occur with pravastatin (metabolism mediated by mechanisms unrelated to CYP3A4). It has been suggested that, when a patient requires coadministration of itraconazol and a statin, the prescription of pravastatin may be preferable instead of atorvastatin (Mazzu et al., 2000).

Concomitant use of statins with warfarin may increase the risk of bleeding, requiring warfarin dose adjustments. The anticoagulant effect of warfarin was shown to be $8-27 \%$ higher in simvastatin-treated patients, probably due to inhibition of CYP2C9 and CYP3A4-dependent warfarin metabolism (Andersson, Eliasson, Lindh, 2012). However, the strength of the interaction varies among individual patients, indicating that polymorphism on CYP2C9 may predispose to drug interaction.

Amiodarone and calcium channel antagonists, such as diltiazem, are inhibitors of CYP3A4. Diltiazem may increase up to four times the plasma concentration of simvastatin (Bellosta, Paoletti, Corsini, 2004).

A randomized trial that determined whether the daily use of simvastatin $80 \mathrm{mg}$ produced greater benefits than $20 \mathrm{mg}$ daily in patients with previous myocardial infarction (SEARCH) revealed an association of myopathy with $80 \mathrm{mg}$ of simvastatin daily and the concomitant use of amiodarone $(\mathrm{RR}=8.8)$. Consequently, patients taking amiodarone were given $20 \mathrm{mg}$ of simvastatin daily, and treatment with amiodarone is now contraindicated with higher doses of simvastatin (Link et al., 2008).

Interaction between statins and fibrates used to be considered a class-effect, until pharmacologic differences between gemfibrozil and fenofibrate were demonstrated. Both fenofibrate and gemfibrozil are metabolized by different families of hepatic glucuronidation enzymes. Fenofibrate is metabolized by uridine diphosphateglucuronosyltransferase (UGT) 1A9 and UGT2B7, whereas gemfibrozil is conjugated by UGT1A1 and UGT1A3, the same family responsible for statin glucuronidation. Thus, gemfibrozil interferes with conversion of statins into its lactone form, necessary for its metabolism via CYP3A4. Moreover, gemfibrozil is a more potent inhibitor of CYP2C9 and CYP2C 8 than fenofibrate (Corsini, Bellosta, Davidson, 2005). In this work, four patients were using fenofibrate concomitantly with simvastatin, and only one had elevated liver enzymes.

Adverse events can compromise patient compliance to treatment, as it has been previously reported (Birtcher, 2015; Chowdhury et al., 2013). Low adherence may be an obstacle to achieve desired clinical outcomes for patients, generating costs for the healthcare system
(Chowdhury et al., 2013). Identification and control of risk factors to reduce the occurrence of adverse events, and, consequently, increase adherence, is, therefore, essential for patient treatment.

This study has some limitations. It is a retrospective study based on data obtained from medical records, which are not fully computerized. Although this aspect did not affect the medical decisions regarding the evaluation or treatment of the patients, it introduced difficulties in data collection. The period and reason for switching or suspending statins were the most difficult sort of information to obtain, together with the knowledge of which medications the patients were using at the time of the change in therapy. Statin dosing and patient adherence to therapy could not also be evaluated.

\section{CONCLUSION}

This study analyzed the risk factors associated with adverse muscular events in dyslipidemic patients treated with statins. The results suggest that the type of statin prescribed and the concomitant use of ABCB1 inhibitors influence the susceptibility to statin-related adverse muscular events.

\section{ACKNOWLEDGMENTS}

We are grateful to the Pharmacy Service at the University Hospital, University of Sao Paulo, Brazil, for the opportunity to develop this study and to participate in the Clinical Pharmacy and Pharmaceutical Care Residency Program (CPPCRP). PFC was a recipient of a CPPCRP scholarship from Ministry of Education, Brazil. RDCH is a recipient of a fellowship from $\mathrm{CNPq}$, Brazil.

\section{REFERENCES}

Ahmad Z. Statin intolerance. Am J Cardiol. 2014;113(10):176571.

Andersson ML, Eliasson E, Lindh JD. A clinically significant interaction between warfarin and simvastatin is unique to carriers of the CYP2C $9 * 3$ allele. Pharmacogenomics. 2012;13(7):757-62.

Armitage J. The safety of statins in clinical practice. Lancet. 2007;370(9601):1781-90.

Bellosta S, Paoletti R, Corsini A. Safety of statins: focus on clinical pharmacokinetics and drug interactions. Circulation. 2004;109(23 Suppl 1):III50-7. 
Birtcher K. When Compliance is an issue-how to enhance statin adherence and address adverse effects. Curr Atheroscler Rep. 2015;17(1):471.

Bitzur R, Cohen H, Kamari Y, Harats D. Intolerance to statins: mechanisms and management. Diabetes Care. 2013;36(2):32530 .

Bonfim MR, Acary SBO, Amaral SL, Monteiro HL. Caracterização do tratamento medicamentoso com estatinas em unidade básica de saúde. 2013;46(14):47-55.

Brasil. Ministério da Saúde. Portaria n²982, de 26 de novembro de 2009. Aprova as normas de execução e de financiamento da assistência farmacêutica na atenção básica. Diário Oficial da União 30 nov 2009; Seção 1.

Brasil. Ministério da Saúde. Portaria nº1554, de 30 de julho de 2013. Dispõe sobre as regras de financiamento e execução do componente especializado da assistência farmacêutica no âmbito do Sistema Único de Saúde. Diário Oficial da União 31 jul 2013.

Bruckert E, Hayem G, Dejager S, Yau C, Bégaud B. Mild to moderate muscular symptoms with high-dosage statin therapy in hyperlipidemic patients--the PRIMO study. Cardiovasc. Drugs Ther. 2005;19(6):403-14.

Carvalho MFC, Romano-Lieber NS, Bergsten-Mendes G, Secoli SR, Ribeiro E, Lebrão ML, et al. Polypharmacy among the elderly in the city of São Paulo, Brazil - SABE Study. Rev Bras Epidemiol. 2012;15(4):817-27.

Chowdhury R, Khan H, Heydon E, Shroufi A, Fahimi S, Moore C, et al. Adherence to cardiovascular therapy: a metaanalysis of prevalence and clinical consequences. Eur Heart J. 2013;34(38):2940-8.

Corsini A, Bellosta S, Davidson MH. Pharmacokinetic interactions between statins and fibrates. Am J Cardiol. 2005;96(9):44-9.

Dean A, Sullivan K, Soe M. OpenEpi: open source epidemiologic statistics for public health, version 3.03a. [Internet]. 2015 [cited 2015 Feb 6]. Available from: www.OpenEpi.com.

Eckel RH. Approach to the patient who is intolerant of statin therapy. J Clin Endocrinol Metab. 2010;95(5):2015-22.

Holtzman CW, Pharm D, Wiggins BS, Spinler SA. Role of P-glycoprotein in statin drug interactions. Pharmacotherapy. 2006;26(11):1601-7.
Instituto Brasileiro de Geografia e Estatísitca. IBGE. Síntese de indicadores sociais - uma análise das condições de vida da população brasileira. Rio de Janeiro: Instituto Brasileiro de Geografia e Estatística; 2013. 266 p.

Institute of Medicine. Promoting cardiovascular health in the developing world: a critical challenge to achieve global health. Washington: National Academy Press; 2010. 482 p.

Jacobsen W, Kirchner G, Hallensleben K, Mancinelli L, Deters M, Hackbarth I, et al. Small intestinal metabolism of the 3-hydroxy-3-methylglutaryl-coenzyme A reductase inhibitor lovastatin and comparison with pravastatin. J Pharmacol Exp Ther. 1999;291(1):131-9.

Jose J. Statins and its hepatic effects: newer data, implications and changing recommendations. J Pharm Bioallied Sci. 2016;8(1):23-8.

Kalliokoski A, Niemi M. Impact of OATP transporters on pharmacokinetics. Br J Pharmacol. 2009;158(3):693-705.

Lexicomp. Drug information handbook: a clinically relevant resource for all healthcare professionals (Lexi-Comp's drug reference handbooks). 23 ${ }^{\text {rd }}$. ed. Hudson: Lexicomp; 2014.

Liao JK. Isoprenoids as mediators of the biological effects of statins. J Clin Invest. 2002;110(3):285-8.

Link E, Parish S, Armitage J, Bowman L, Heath S, Matsuda F, et al. SLCO1B1 variants and statin-induced myopathy - a genomewide study. N Engl J Med. 2008;359(8):789-99.

Mammen AL, Amato AA. Statin myopathy: a review of recent progress. Curr Opin Rheumatol. 2010;22(6):644-50.

Mancini GBJ, Tashakkor AY, Baker S, Bergeron J, Fitchett D, Frohlich J, et al. Diagnosis, prevention, and management of statin adverse effects and intolerance: Canadian working group consensus update. Can J Cardiol Canadian Cardiovascular Society; 2013;29(12):1553-68.

Mazzu AL, Lasseter KC, Shamblen EC, Agarwal V, Lettieri J, Sundaresen P. Itraconazole alters the pharmacokinetics of atorvastatin to a greater extent than either cerivastatin or pravastatin. Clin Pharmacol Ther. 2000;68(4):391-400.

Minder CM, Blumenthal RS, Blaha MJ. Statins for primary prevention of cardiovascular disease : the benefits outweigh the risks. Curr Opin Cardiol. 2013;28(5):554-60. 
Oh J, Ban MR, Miskie BA, Pollex RL, Hegele RA. Genetic determinants of statin intolerance. Lipids Health Dis. 2007;6(1):7-11.

Reiner Ž. Resistance and intolerance to statins. Nutr Metab Cardiovasc Dis. 2014;24(10):1057-66.

Russo MW, Hoofnagle JH, Gu J, Fontana RJ, Barnhart H, Kleiner DE, et al. Spectrum of statin hepatotoxicity: experience of the drug-induced liver injury network. Hepatology. 2014;60(2):679-86.

Russo MW, Scobey M, Bonkovsky HL. Drug-induced liver injury associated with statins. Semin Liver Dis. 2009;29(4):41222.

Shi S, Klotz U. Age-related changes in pharmacokinetics. Curr Drug Metab. 2011;12(7):601-10.
Thai M, Reeve E, Hilmer SN, Qi K, Pearson S-A, Gnjidic D. Prevalence of statin-drug interactions in older people: a systematic review. Eur J Clin Pharmacol. 2016;72(5):513-21.

Truven Health Analytic. Micromedex Drug Information [Internet]. [cited 2015 Jan 30]. Available from: http://www. micromedexsolutions.com/micromedex2/librarian/.

Viktil KK, Blix HS, Reikvam $\AA$. The Janus face of polypharmacy - overuse versus underuse of medication. Nor Epidemiol. 2008;18(2):147-52.

Xavier HT, Izar MC, Faria Neto JR, Assad MH, Rocha VZ, Sposito AC, et al. V diretriz brasileira de dislipidemias e prevenção da aterosclerose. Arq Bras Cardiol. 2013;101(4 Suppl 1):1-22.

Received for publication on $27^{\text {th }}$ November 2016 Accepted for publication on $07^{\text {th }}$ August 2017 